\title{
AN APPROACH TO FORENSIC FACE RECOGNITION
}

\author{
ANGADI S.A. ${ }^{1}$, HATTURE S.M. ${ }^{2}$ AND KARCHI R.P. ${ }^{3}$ \\ 1,2Department of Computer Science and Engineering, Basaveshwar Engineering College, Bagalkot, Karnataka State, India. \\ ${ }^{3}$ Department of Computer Applications, Basaveshwar Engineering College, Bagalkot, Karnataka State, India. \\ *Corresponding Author: Email-1 vinay_angadi@yahoo.com, 2smhatture@yahoo.com and 3rashmi_karchi@rediffmail.com
}

Received: April 12, 2012; Accepted: May 15, 2012

\begin{abstract}
For criminal investigators in the field of forensic science the face recognition is challenging task. As the forensic face images captured under non-ideal conditions with complex background and variation in the pose. This paper is implemented PCA and Eigenface method for face recognition with variation in the pose. The purpose of PCA is to reduce the large dimensionality of the facial data space to the smaller intrinsic dimensionality of feature space. The face images are projected onto face space simply by multiplying the difference between the image and the average and the result is multiplied by each eigenvector. The result of this operation will be the weighted contribution of each Eigenface in representing the input face image, treating the Eigenfaces as a basis set for face images. The Euclidean distance taken from the features of captured face image and available facial features will determine the matching of the face image. The Principal Component Analysis (PCA) using Eigenface method is an efficient method for face recognition in the field of forensic science. The experimental results shows that the proposed method is best suited for face recognition with variation in the pose.
\end{abstract}

Keywords- Forensic science, Face recognition, PCA, Eigenface, Feature space, Euclidean distance

Citation: Angadi S.A., Hatture S.M. and Karchi R.P. (2012) An Approach to Forensic Face Recognition. International Journal of Machine Intelligence, ISSN: 0975-2927 \& E-ISSN: 0975-9166, Volume 4, Issue 1, pp.-403.

Copyright: Copyright@2012 Angadi S.A., Hatture S.M. and Karchi R.P. This is an open-access article distributed under the terms of the Creative Commons Atribution License, which permits unrestricted use, distribution, and reproduction in any medium, provided the original author and source are credited. 